\title{
CDKN2A deletion is a frequent event associated with poor outcome in patients with peripheral T-cell lymphoma not otherwise specified (PTCL-NOS)
}

Haematologica 2021

Volume 106(11):2918-2926

\section{Correspondence: \\ PAOLO CORRADINI \\ paolo.corradini@unimi.it}

FRANCESCO MAURA

francesco.maura85@gmail.com

Received: June 12, 2020.

Accepted: September 2, 2020.

Pre-published: September 10, 2020.

https://doi.org/10.3324/haematol.2020.262659

(C)2021 Ferrata Storti Foundation

Material published in Haematologica is covered by copyright. All rights are reserved to the Ferrata Storti Foundation. Use of published material is allowed under the following terms and conditions:

https://creativecommons.org/licenses/by-nc/4.0/legalcode. Copies of published material are allowed for personal or internal use. Sharing published material for non-commercial purposes is subject to the following conditions:

https://creativecommons.org/licenses/by-nc/4.0/legalcode, sect. 3. Reproducing and sharing published material for commercial purposes is not allowed without permission in writing from the publisher.
Francesco Maura, ${ }^{1-4}$ Anna Dodero, ${ }^{5}$ Cristiana Carniti, ${ }^{5}$ Niccolò Bolli,,${ }^{2,5}$ Martina Magni, ${ }^{5}$ Valentina Monti, ${ }^{6}$ Antonello Cabras, ${ }^{6}$ Daniel Leongamornlert, ${ }^{3}$ Federico Abascal, ${ }^{3}$ Benjamin Diamond, ${ }^{1}$ Bernardo Rodriguez-Martin, ${ }^{7}$ Jorge Zamora, ${ }^{7}$ Adam Butler, ${ }^{3}$ Inigo Martincorena, ${ }^{3}$ Jose M. C. Tubio, ${ }^{7}$ Peter J. Campbell, ${ }^{3}$ Annalisa Chiappella, ${ }^{8^{\circ}}$ Giancarlo Pruneri ${ }^{2,6}$ and Paolo Corradini ${ }^{2,5}$

${ }^{1}$ Myeloma Service, Department of Medicine, Memorial Sloan Kettering Cancer Center, New York, NY, USA; ${ }^{2}$ Department of Oncology and Hemato-Oncology, University of Milan, Milan, Italy; ${ }^{3}$ The Cancer, Aging and Somatic Mutation Program, Wellcome Sanger Institute, Hinxton, Cambridgeshire, UK; ${ }^{4}$ Weill Cornell Medical College, New York, NY, USA; ${ }^{5}$ Department of Medical Oncology and Hematology, Fondazione IRCCS Istituto Nazionale dei Tumori, Milan, Italy; ${ }^{6}$ Department of Pathology and Laboratory Medicine, Fondazione IRCCS Istituto Nazionale dei Tumori, Milan, Italy; ${ }^{7} \mathrm{CIMUS}$ - Molecular Medicine and Chronic Diseases Research Center, University of Santiago de Compostela, Santiago de Compostela, Spain and ${ }^{8}$ Department of Hematology Azienda Ospedaliera Città della Salute e della Scienza, Turin, Italy.

${ }^{\circ}$ Current address: Department of Medical Oncology and Hematology, Fondazione IRCCS Istituto Nazionale dei Tumori, Milan, Italy.

\section{ABSTRACT}

Todal peripheral T-cell lymphoma not otherwise specified (PTCLNOS) remains a diagnosis encompassing a heterogenous group of They are characterized by a poor clinical outcome when treated with anthracycline-containing regimens. A better understanding of their biology could improve prognostic stratification and foster the development of novel therapeutic approaches. Recent targeted and whole exome sequencing studies have shown recurrent copy number abnormalities (CNA) with prognostic significance. Here, investigating five formalinfixed, paraffin embedded cases of PTCL-NOS by whole genome sequencing, we found a high prevalence of structural variants and complex events, such as chromothripsis likely responsible for the observed CNA. Among them, CDKN2A and PTEN deletions emerged as the most frequent aberration, as confirmed in a final cohort of 143 patients with nodal PTCL. The incidence of CDKN2A and PTEN deletions among PTCL-NOS was $46 \%$ and $26 \%$, respectively. Furthermore, we found that co-occurrence of CDKN2A and PTEN deletions is an event associated with PTCLNOS with absolute specificity. In contrast, these deletions are rare and never co-occur in angioimmunoblastic and anaplastic lymphomas. CDKN2A deletion was associated with shorter overall survival in multivariate analysis corrected by age, International Prognostic Index, transplant eligibility and GATA3 expression (adjusted Hazard Ratio $=2.53$; 95\% Confidence Interval: 1.006-6.3; $P=0.048$ ). These data suggest that CDKN2A deletions may be relevant for refining the prognosis of PTCLNOS and their significance should be evaluated in prospective trials.

\section{Introduction}

Within the heterogeneous categories of nodal peripheral T-cell lymphoma (PTCL) subtypes currently recognized by the World Health Organization (WHO) classification system, ${ }^{1-4}$ many subtypes have defining molecular and phenotypic features. For example, within the anaplastic large cell lymphoma (ALCL) subtype, translocations 
of the $A L K$ oncogene define a homogeneous subgroup, while ALK-negative cases carry mutations or translocations resulting in the activation of the JAK/STAT pathway. ${ }^{5}$ In angioimmunoblastic T-cell lymphoma (AITL), mutations in DNMT3A, TET2, IDH2 and RHOA have been described in a significant fraction of patients..$^{6-8}$ These features are increasingly used for diagnostic and prognostic purposes, and may translate into targeted or rationale treatment approaches. ${ }^{9,10}$ A subgroup of PTCL not otherwise specified (PTCL-NOS) carries a T-cell follicular helper (TFH) phenotype and some phenotypic and genetic features of AITL including mutations affecting TET2, DNMT3A, and RHOA genes. ${ }^{6,11}$ Therefore, nodal PTCL with TFH phenotype have been included in the revised WHO classification as a provisional entity. ${ }^{4}$ On the contrary, the vast majority of PTCLNOS are a large yet ill-defined subgroup that lack defining genetic of phenotypic features. ${ }^{6,11-13}$ They are characterized by a poor clinical outcome when treated with anthracycline-containing regimens and some non-randomized studies suggest that high-dose chemotherapy can offer some survival benefit in young patients. ${ }^{14,15}$ However, a better understanding of their biology could improve prognostic stratification and foster the development of novel therapeutic approaches.

The gene expression profile of PTCL-NOS suggests that two major subtypes can be identified, one characterized by GATA3 and the other by TBX21 overexpression, the former carrying worse prognosis. ${ }^{16-18}$ More recently, two groups identified recurrent copy-number alterations (CNA) in the tumor suppressors TP53 and CDKN2A with adverse prognostic significance, and generally associated with genomic instability. ${ }^{19,20}$ These efforts have been based on next-generation sequencing (NGS) of targeted gene panels and singlenucleotide polymorphism (SNP) arrays. Contrary to the above studies, whole genome sequencing (WGS) can interrogate the full repertoire of somatic mutations, CNA, structural variants (SV) and even mutational processes involved in cancer pathogenesis. ${ }^{21}$ However, WGS in PTCL have been hampered by the availability of high-quality, tumorrich DNA. On the other end, novel findings must be validated and applicable to everyday diagnostic routine before the benefits of additional information are translated into clinical improvements.

With the hypothesis that WGS may reveal the genomic bases of recurrent genomic alterations in PTCL-NOS, here we decided to test this approach in formalin-fixed, paraffin embedded (FFPE) samples. Furthermore, using an extended cohort of cases, we validate our and published results by fluorescence in situ hybridization (FISH) and immunohistochemistry (IHC), and analyze prognostic correlates.

\section{Methods}

\section{Sample selection}

Eleven PTCL-NOS patients treated at the Department of Hematology, Fondazione IRCCS Istituto Nazionale dei Tumori, Milan, Italy were selected for the WGS analysis (Online Supplementary Table S1). The study protocol was approved by the Institutional Review Board (n. INT 19/13) and was conducted in accordance with the Declaration of Helsinki. The diagnosis was made according to the 2016 WHO classification ${ }^{4}$ and subtype assignment was independently reviewed by two expert hemato-pathologists. Only PTCL-NOS cases were selected. Nodal lymphomas with TFH cell origin, defined according to the 2017 WHO update 4 by the association of a CD4+ phenotype with the expression of at least two TFH markers among ICOS, PD1, CXCL13, BCL-6 and CD10 by, were excluded on morphology and immunohistochemical bases. Additionally a comprehensive morphologic assessment was performed evaluating features commonly associated with nodal lymphomas with TFH cell origin such as the presence of large Reed-Sternberg-like, CD30+/CD15+ and PAX5+ cells, Eppstein Bar virus (EBV) positivity without a pabulum and with a growth pattern that mimics either a follicular lymphoma or progressively transformed germinal centers. Samples were chosen according to tumor cellularity and amount of extracted DNA ( $>500 \mathrm{ng}$ ). DNA was extracted from FFPE blocks in ten patients, as previously described. ${ }^{22}$ For one patient (PD30774a), DNA was extracted from a fresh frozen sample (peripheral blood mononuclear cells harvested during leukemic progression). For one patient, we sequenced two samples: one collected at diagnosis (PD30770a) and one at relapse (PD30770c). Therefore, all but two samples (PD30774a and PD30770c) were collected at diagnosis. DNA from buccal swabs was used as normal match for all samples.

\section{Whole genome sequencing data analysis}

WGS libraries were prepared with the TruSeq DNA PCR-Free Library Preparation Kit (Illumina San Diego, CA, USA) from 500 ng of genomic DNA, aiming for an average target insert of 300

Table 1. Demographic and clinical characteristics of the peripheral T-cell lymphoma not otherwise specified cohort used for survival analysis.

\begin{tabular}{|c|c|c|c|c|c|}
\hline Variable & All patients & CDKN2 deleted & CDKN2 wt & $n / e$ & P-value \\
\hline Age (range) & $59.9(22-85)$ & $59.5(22-84)$ & $59.9(34-85)$ & & 0.7 \\
\hline Sex (female) & $17 / 34(50 \%)$ & $7 / 20(35 \%)$ & 10/14 (71\%) & 0 & 0.07 \\
\hline Bone Marrow infiltration & $9 / 30(30 \%)$ & $4 / 18(22 \%)$ & $5 / 12(41 \%)$ & 4 & 0.4 \\
\hline Extra-nodal disease & 12/34 (35\%) & $9 / 20(45 \%)$ & $3 / 14(21 \%)$ & 0 & 0.27 \\
\hline IPI $>2$ & 14/32 (43\%) & 7/18 (39\%) & 8/14 (57\%) & 2 & 0.47 \\
\hline SCT eligible & 27/34 (79\%) & $16 / 20(80 \%)$ & 11/14 (78\%) & 0 & 1 \\
\hline $\begin{array}{l}\text { Anthracycline } \\
\text { induction CT }\end{array}$ & $31 / 34(91 \%)$ & $18 / 20(90 \%)$ & $13 / 14(9 \%)$ & 0 & 0.7 \\
\hline Response to first linel (CR) & 13/33 (39\%) & $5 / 20(25 \%)$ & $5 / 13(38 \%)$ & 1 & 0.06 \\
\hline ASCT or AlloSCT & $14 / 34(41 \%)$ & $5 / 20(25 \%)$ & $9 / 14(64 \%)$ & 0 & 0.035 \\
\hline GATA3 >50\% & $9 / 34(26 \%)$ & $6 / 20(30 \%)$ & $3 / 14(21 \%)$ & 0 & 0.7 \\
\hline
\end{tabular}

SCT: stem cell transplantation; ASCT: autologous stem cell transplantation; AlloSCT: allogeneic stem cell transplantation; CR: complete remission; PT: partial remission; n/e: not evaluable; IPI: International Prognostic Index; wt: wild-type; CT: chemotherapy. 
basepairs (bp). Sequencing was performed on the Illumina X10 platform at the Wellcome Sanger Institute (WSI) on a $150 \mathrm{bp}$ paired end protocol to a target depth of 40x for tumor samples and 30x for normal controls. The sequence data described here will be made available from the European Genome-phenome Archive (EGA) repository (EGAS00001002057). FASTQ files were aligned to the reference genome (GRCh37/hg19) using BWAmem and deduplicated aligned BAM files were analyzed using the following published tools available at the WSI: ASCAT and Battenberg to measure clonal and subclonal CNA and to estimate the tumor cell fraction of each sample, ${ }^{23}$ Caveman and Pindel for single nucleotide variants (SNV) and small insertion-deletions (indels); ${ }^{24,25}$ BRASS for SV (large inversions and deletions, translocations, internal tandem duplications). ${ }^{23}$ Complex events were defined, as previously described. ${ }^{26}$ TraFIC was used to described the somatic L1 retrotransposition landscape..$^{27}$

The repertoire of mutational processes operative in PTCLNOS was analyzed by extracting the corresponding mutational signatures using the 96-class matrix of all possible substitutions in their 5' and 3' context with non-negative matrix factorization (NNMF), as previously described..$^{28,29}$ Mutations were classified as drivers based on the COSMIC census catalogue of cancer genes (http://cancer.sanger.ac.uk/cosmic/).

\section{Validation cohorts}

In order to expand our analysis on mutations in driver genes, we included published exomes data from 63 PTCL patients (30 PTCL-NOS ,15 AITL, 23 ALCL ALK neg and 16 EATL-II ). $6,7,5,30,31,13$ COSMIC census was used to create the catalogue of genes potentially involved by nonsynonymous mutations (https://cancer.sanger.ac.uk/census).

CDKN2A and PTEN status was validated by dual-colour FISH on paraffin-embedded selected tumor areas using commercially available DNA probes: for p16 (CDKN2A) we used a spectrum orange-labeled locus-specific CDKN2A (9p21) probe and spectrum green-labeled chromosome 9 centromeric probe (LSI CDKN2A/CEP 9); for PTEN, a spectrum orange-labeled locusspecific PTEN (10q23) probe and a spectrum green-labeled chromosome 10 centromeric probe (LSI PTEN/CEP 10) - Vysis Inc., Downers Grove, IL, USA. A CDKN2A deletion was defined in the presence of either a homozygous deletion in $>10 \%$ of cells or a hemizygous deletion in $>40 \%$ of cells. ${ }^{27} \mathrm{~A}$ cutoff or $40 \%$ was applied to define the hemizygous PTEN loss. CDKN2A/p16 and GATA3 protein expression was tested by IHC, as previously described. ${ }^{16-18}$ In order to define GATA3 IHC positivity we used the recently proposed $50 \%$ cutoff. ${ }^{18}$

For further validation, we included published data from 20 AITL samples with copy number status defined using different NGS approach ${ }^{7,31}$ and 81 PTCL investigated by SNP array. ${ }^{32,33}$ For this last series, we downloaded all the available CEL files from GEO (accession numbers GSE15842 and GSE50252) and then $\operatorname{LogR}$ and BAF data were extracted using pennCNV. ${ }^{34}$ We then applied the ASCAT algorithm to perform segmentation and retrieve allele-specific absolute copy number alterations. The description of entire cohort used to validate CDKN2A and PTEN status can be found in the Online Supplementary Table S2.

All contingency analyses were performed by Fisher's exact test. Estimated progression-free survival (PFS) and overall survival (OS) were calculated with the Kaplan-Meier method and groups were compared with the log-rank test. Multivariate analysis was performed with Cox regression. All the analyses were performed using appropriate functions in the R 3.4.2 software (wWw.r-project.org).

\section{Results}

\section{Peripheral T-cell lymphoma not otherwise specified show a complex genomic architecture}

We performed WGS on 12 tumors and 11 matched normal samples from 11 patients affected by PTCL-NOS, achieving an average depth of $27 x$ (Online Supplementary Table S1). For one patient (PD30770) a diagnosis and a relapse tumor sample were available. As expected, analysis was severely hampered by the nature of samples analyzed, so that samples from six patients did not pass quality control. Causes ranged from insufficient cluster generation due to low quality DNA (one sample), low cancer cell fraction (CCF) (one sample), FFPE-induced artifacts (four samples). More data on quality control, FFPE artifacts and mutational signatures in PTCL-NOS WGS samples can be found in the Online Supplementary Appendix section.

In the remaining five patients, no recurrent point mutations or indels in onco-driver genes were identified, in line with recent observations. ${ }^{6,11}$ The rare involvement of oncodriver gene in PTCL-NOS compared to other PTCL subtypes was confirmed in additional 84 published WES cases ${ }^{5-}$ 7,30,31 (Online Supplementary Figure S1-2). We therefore focused the investigation on structural variants (SV: defined as inversions, translocations, internal tandem duplications [ITD] and deletions) and CNA, which could be investigated in depth in our five WGS samples. Three hundred and seventy-two SV were extracted with a median of 74 per sample (range, 56-86) (Figure 1A). Intriguingly, at least one complex event was observed in all but one patient, including five chromothripsis events in three patients (Figure 1B). ${ }^{35}$ Importantly, several cancer genes were affected either by SV directly or by CNA caused by SV and complex events (Figure 1C). For example, sample PD30771a was characterized by three different chromothripsis events on multiple chromosomes, including one causing a homozygous deletion of CDKN2A (Figure 1C). The genomic landscape was even more complex in relapse samples: PD30774a showed a whole genome duplication, and a large chromothripsis event was responsible for disruption of several known oncogenes resulting, for instance, in $A R I D 1 B$ and $A R I D 2$ losses (Figure 1C); analysis of serial diagnosis and relapse samples in patient PD30770 showed acquisition of numerous SV and CNA, and a complex event in chromosome 16 (Online Supplementary Figure S3). Overall, this confirms how driver events in PTCL-NOS may often go beyond coding gene mutations and involve complex structural events that can only be investigated by whole genome sequencing.

\section{CDKN2A and PTEN are frequently co-deleted in peripheral T-cell lymphoma not otherwise specified, but not in other peripheral T-cell lymphoma}

Most of CNA and SV breakpoints were not recurrent, but we found the tumor suppressor CDKN2A deleted in four out of five patients. Collectively, we found a simple homozygous deletion (HD), and a second HD caused by chromothripsis. In two further cases, breakpoints around the copy-number loss could not be resolved by a distinct SV event, so the cause remains unknown (Figure 1C). Interestingly, PTEN loss was observed in two out of four CDKN2A-deleted patients. FISH analysis on sections from the five cases analyzed by WGS confirmed the CDKN2A and the PTEN deletion in all cases (Figure 2A), and the diploid status of the genes in the ones where no deletions 
were found by NGS. The high prevalence of these deletions in our small dataset confirmed recently published data and prompted us to extend our observations. To this end, we evaluated by FISH 37 PTCL archive cases from our institution (30 PTCL-NOS, five ALK negative ALCL and two AITL cases, Online Supplementary Table S2). The prevalence of
CDKN2A deletions among PTCL-NOS was $50 \%$, and $\mathrm{HD}$ accounted for two thirds of cases. Overall, $31 \%$ of cases showed PTEN deletions.

In order to compare the prevalence of CDKN2A and PTEN deletion across the main PTCL histologies, we included 101 cases from published datasets ${ }^{7,31-33}$ for a total of
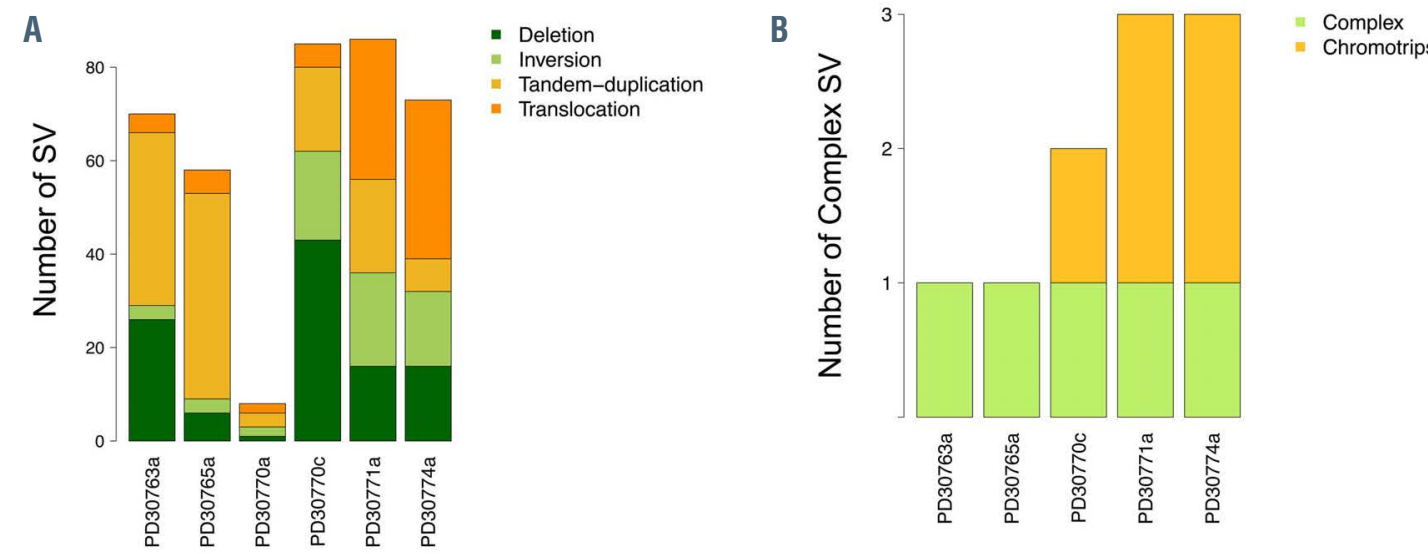

C Deletion Translocation Other Complex Chromothripsis Not determinable

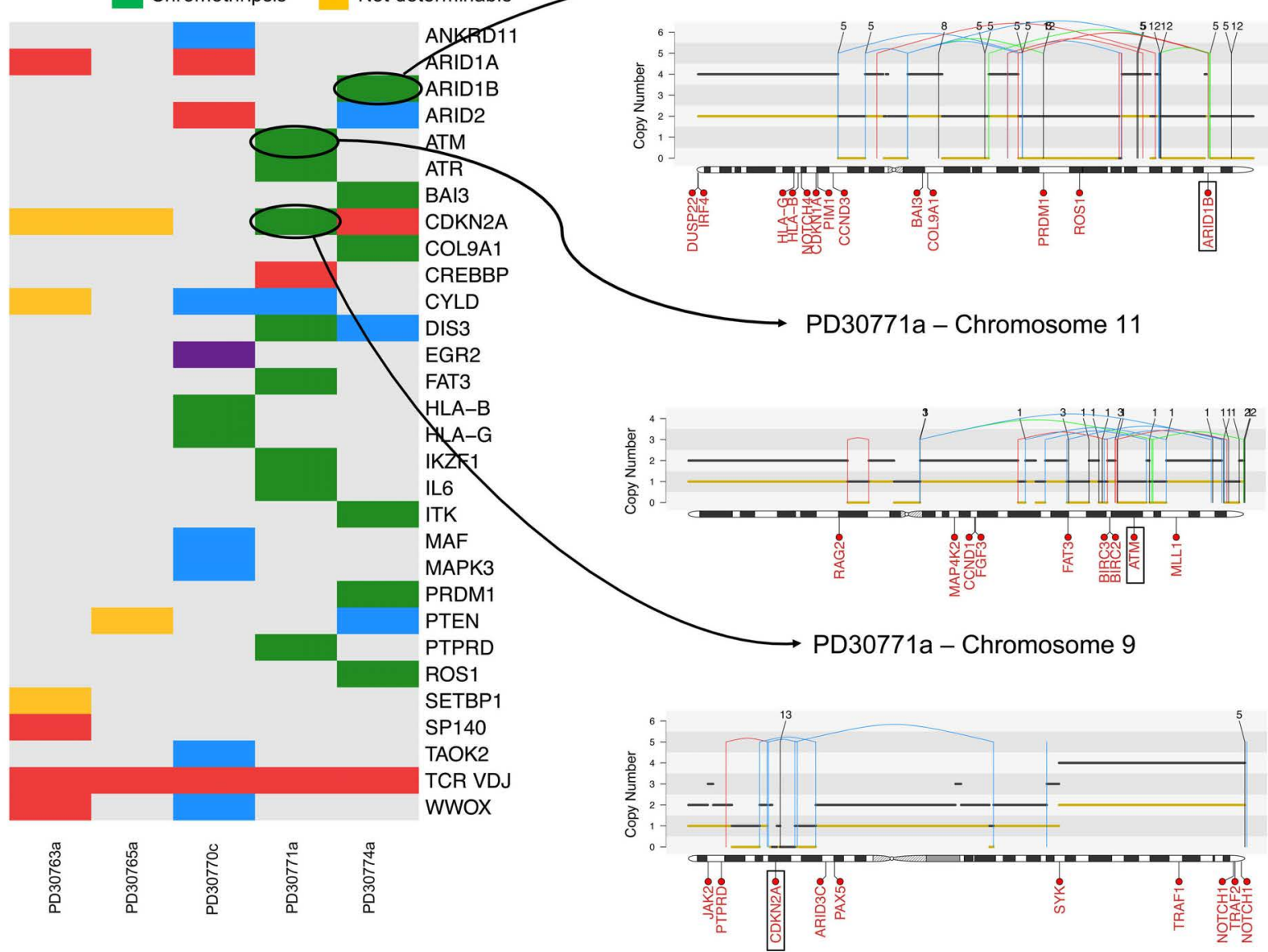

Figure 1. Peripheral T-cell lymphoma not otherwise specified structural variants and complex events. Prevalence of structural variants (A) and complex events (B) among peripheral T-cell lymphoma not otherwise specified (PTCL-NOS). (C) Heatmap summarizing the oncogenes whose disruption was caused by at least one structural variants. The heatmap color highlighted the type of structural variant (SV) involved. Yellow: not determinable, i.e., the oncogene loss could not be mapped to a specific SV. For three events, the chromothripsis event responsible for the oncogene loss is drawn on the right. The horizontal black line indicates total copy number; the orange line minor copy number. Vertical lines represent SV breakpoints for deletion (red), inversion (blue), tandem-duplication (green) and translocation (black). 
143 PTCL of which 63 PTCL-NOS (Online Supplementary Table S2; Online Supplementary Figure S4). CDKN2A was deleted in 29 of 63 (46\%) PTCL-NOS cases, including 19 of $29(65 \%) \mathrm{HD}$. This was significantly more frequent than what was found in other histologies (Figure $2 \mathrm{~B}$ and $\mathrm{C}$ ). PTEN was deleted in 17 of 63 (26\%) PTCL-NOS cases, 13 $(76 \%)$ of which also carried a CDKN2A loss. Importantly, the co-occurrence of CDKN2A and PTEN was specific for PTCL-NOS (Figure $2 \mathrm{~B}$ and $\mathrm{C}$ ), representing the first genetic abnormality of such kind.

\section{CDKN2A genetic status does not correlate with p16 nor with GATA3 expression}

We next evaluated CDKN2A expression (p16) by IHC in 31 PTCL-NOS samples for whose archival material was available. Overall, the average percentage of tumor cells expressing p16 in WT cases was $23.44 \%$ (range, $0-90 \%$ ), while it was $6.21 \%$ (range, $0-30 \%$ ) in deleted cases, either mono- or bi-allelic. This difference was significant $(P=0.01$, Student $t$-test) (Figure $3 \mathrm{~A}$ and $\mathrm{C}$ ). Using the previously described cutoff of $20 \%$ of tumor cells to define a categorical present/absent $\mathrm{p} 16$ expression status, only one case was p16 positive when $C D K N 2 A$ was deleted, and no cases of bi-allelic deletions showed p16 positivity. However, nine of 14 cases were p16 negative in spite of a wild-type (WT) CDKN2A locus (Figure 3A). This suggests that reduced expression of $\mathrm{p} 16$ can stem from non-genetic events, including epigenetic or post-transcriptional mechanisms as reported in other cancer types. ${ }^{36,37}$

Next, we correlated the genetic status of CDKN2A and GATA3 expression. Again, the picture was far from clear: the average percentage of GATA3-expressing cells was $23.12 \%$ in CDKN2A WT cases, and $33.72 \%$ in CDKN2A deleted cases. This difference was not significant $(P=0.01$, Student $t$-test) (Figure 3D). Even after setting the threshold for GATA3 expression at $50 \%,{ }^{18}$ the number of GATA3 positive cases did not differ between CDKN2A WT and deleted cases.

Finally, taking advantage of the combined WGS-SNP array cohort of 33 PTCL-NOS cases, we explored additional structural events in this subgroup. We observed $17 \mathrm{p} 13$ (TP53) deletions in 11 patients (33\%), with partial overlap with CDKN2A deletions (Online Supplementary Figure S5).

\section{CDKN2A deletions carry prognostic significance}

Clinical data were available for all PTCL-NOS patients

A

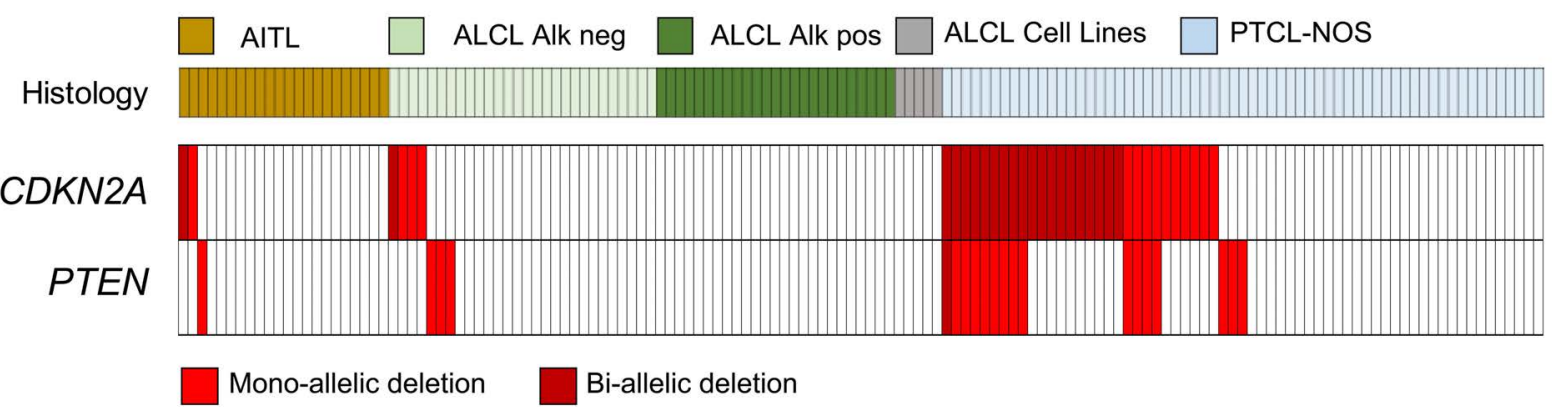

B

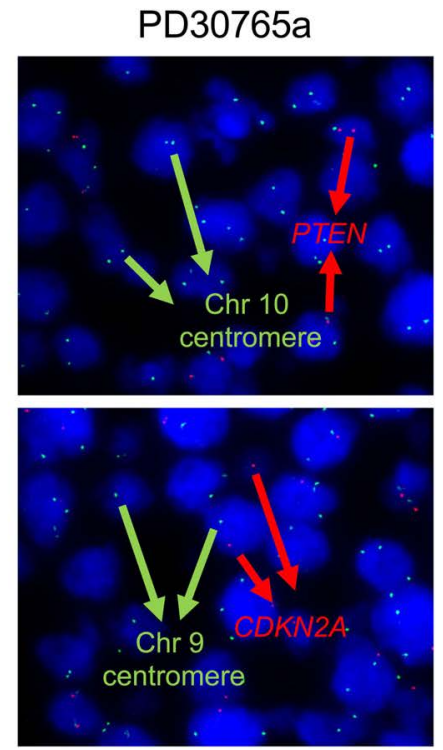

C

\begin{tabular}{cccc}
\hline Histology & $\begin{array}{c}\text { CDKN2A } \\
\text { deletion }\end{array}$ & $\begin{array}{c}\text { CDKN2A bi- } \\
\text { deletion }\end{array}$ & $\begin{array}{c}\text { PTEN and } \\
\text { CDKN2A co- } \\
\text { deletions }\end{array}$ \\
\hline PTCL-NOS & $29 / 63(46 \%)$ & $19 / 63(30 \%)$ & $13 / 63(20 \%)$ \\
\hline ALCL ALK neg & $4 / 28(14 \%)$ & $1 / 28(3.5 \%)$ & $0 / 28(0 \%)$ \\
\hline ALCL ALK pos & $0 / 25(0 \%)$ & $0 / 25(0 \%)$ & $0 / 25(0 \%)$ \\
\hline AITL & $2 / 22(9 \%)$ & $1 / 22(4.5 \%)$ & $0 / 22(0 \%)$ \\
\hline$P$-value & $P<0.01$ & $P<0.01$ & $P<0.01$ \\
\hline
\end{tabular}

Figure 2. Prevalence of CDKN2A and PTEN deletions among different peripheral T-cell lymphoma subtypes. (A) Heatmap showing the prevalence of CDKN2A and PTEN deletions across the main peripheral T-cell lymphoma (PTCL) subtypes. (B) representative fluorescence in situ hybridization (FISH) pictures of PTEN (top) and CDKN2A (bottom) deletions. (C) Frequency table of CDKN2A deletions, mono- and bi-allelic, and co-occurrence with PTEN deletions across PTCL subtypes. All statistical comparisons were performed by Fisher's exact test. Peripheral T-cell lymphoma not otherwise specified (PTCL-NOS) showed a significant enrichment for these three genomic aberrations compared to all the other PTCL histologies $(P<0.01)$. ALCL: anaplastic large cell lymphoma; AITL: angioimmunoblastic T-cell lymphoma neg: negative; pos: positive. 
from our WGS and FISH cohort except one that was not treated as a PTCL-NOS due to an initial diagnostic misclassification. The median age of the cohort was 59 years (range, 22-85), with 79\% (27 of 34) transplant eligible and $43 \%$ (14 of 32 ) with an International Prognostic Index (IPI) $>2$. Most of the patients $(n=31,94.4 \%)$ received an induction therapy with an anthracycline. During the course of the disease, 14 patients (41\%) received autologous stem cell transplantation (SCT) and/or allogeneic SCT (Table 1). ${ }^{38,14}$

After a median follow-up of 70 months, 11 patients $(32 \%)$ were still alive. We thus evaluated whether CDKN2A and PTEN genomic status affected the response to therapy and outcome. PTEN deletion did not impact the clinical outcome in terms of both PFS and OS. In contrast, CDKN2A deleted cases were more frequent in primary refractory than WT cases (15 of 20 [75\%] vs. five of 13 [38\%]; $P=0.06)$. Furthermore, 16 of 20 (80\%) CDKN2Adeleted patients died in disease progression already within the first year of diagnosis. Consequently, PTCL-NOS patients carrying $C D K N 2 A$ deletions had a significantly shorter PFS compared to WT patients (5-year PFS: 7.5\% [95\% Confidence Interval (CI): 1.3-42] vs. 24\%, [95\% CI: 963.5]; $P=0.048$ ) and OS (5-year OS: $22.5 \%$ [95\% CI: 9.4-54] vs. $52 \%$ [95\%CI: 30-90]; $P=0.039$ ) (Figure $4 \mathrm{~A}$ and $\mathrm{B}$ ). The association between short survival and CDKN2A deletion retained its significance after multivariate correction by age, IPI, transplant eligibility and GATA3 IHC expression (Figure $4 \mathrm{C}$ and D).

\section{Discussion}

In this paper we performed the first WGS experiment on PTCL-NOS samples. Despite the limited series, WGS showed ubiquitous instances of complex rearrangements and prevalent inactivation of classical tumor suppressor genes by SV and CNA. Chromothripsis in particular, a common mechanism underlying complex rearrangements, was never described in PTCL-NOS and frequently seen in our samples thanks to the comprehensive analysis of the genome allowed by WGS. This observation likely provides the mechanistic bases of the frequent CNA observed in PTCL-NOS cases in larger cohorts, including CDKN2A deletions. $^{19,20}$ In our study, we could also observe how CDKN2A deletions were often focal and bi-allelic. This inactivation pattern is highly specific for driver tumor suppressors, and has been described for CDKN2A in other hematological malignancies. ${ }^{39-41}$ We went on to confirm this

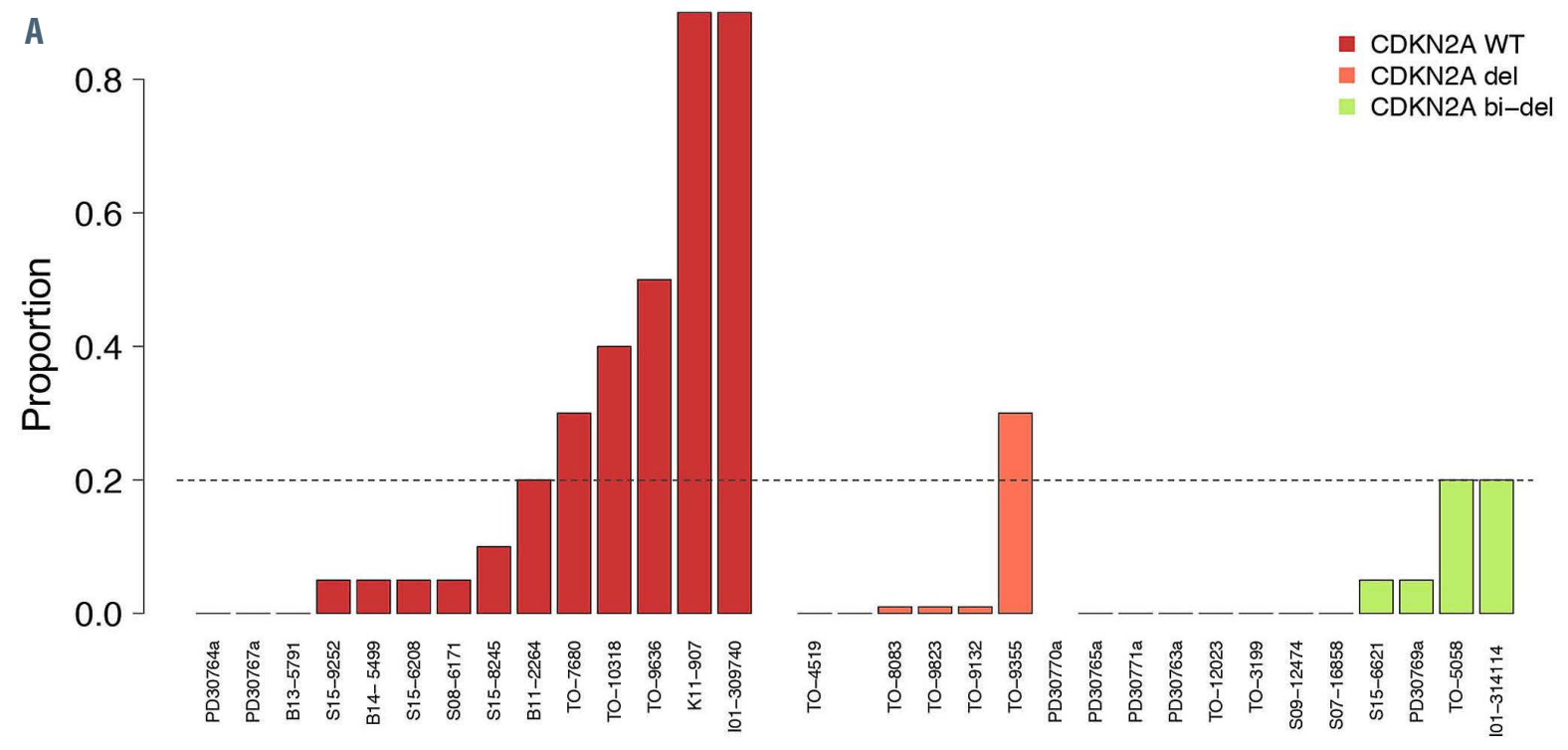

B

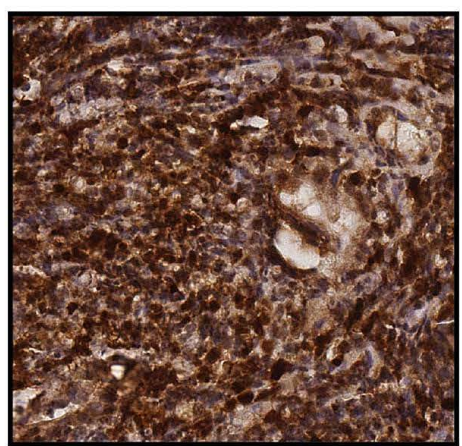

C

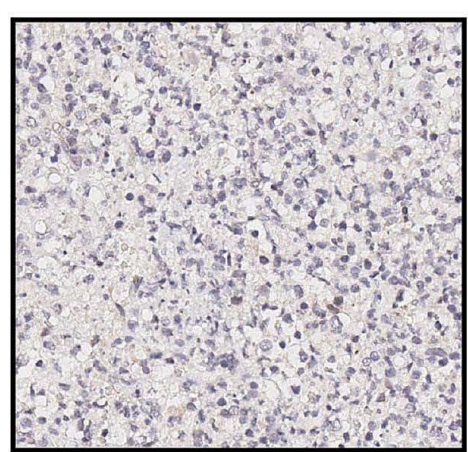

D

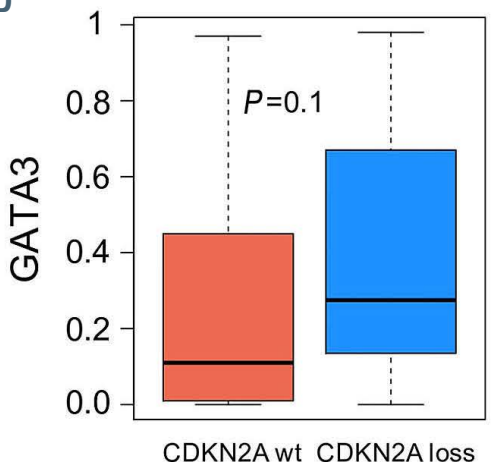

Figure 3. CDKN2A protein expression in peripheral T-cell lymphoma not otherwise specified. (A) Expression of CDKN2A/p16 by immunohistochemistry (IHC). (B and C) Representative examples of CDKN2/p16 IHC results in cases with normal (B) and deleted (C) alleles. (D) GATA3 protein expression evaluated by IHC in CDKN2A deleted (del) and wild-type (wt) cases. P-value was estimated using a Wilcoxon test. 
A

Progression-free survival

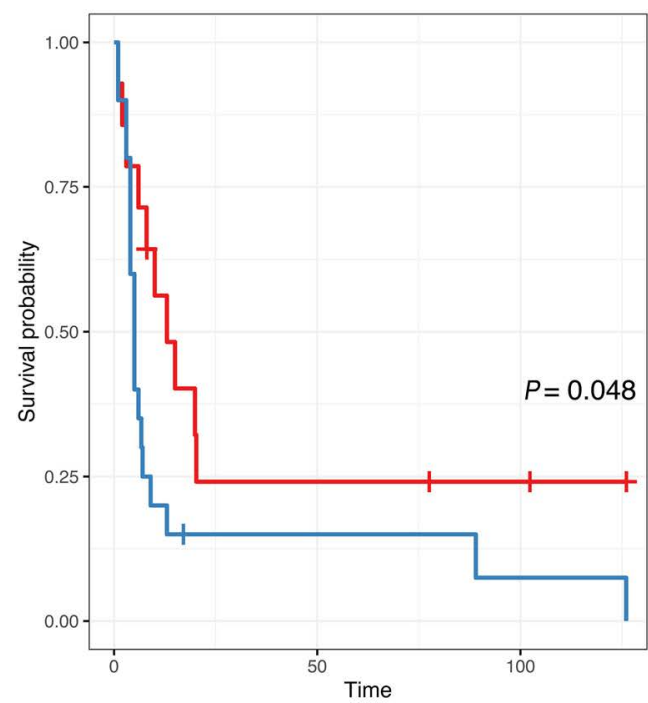

C

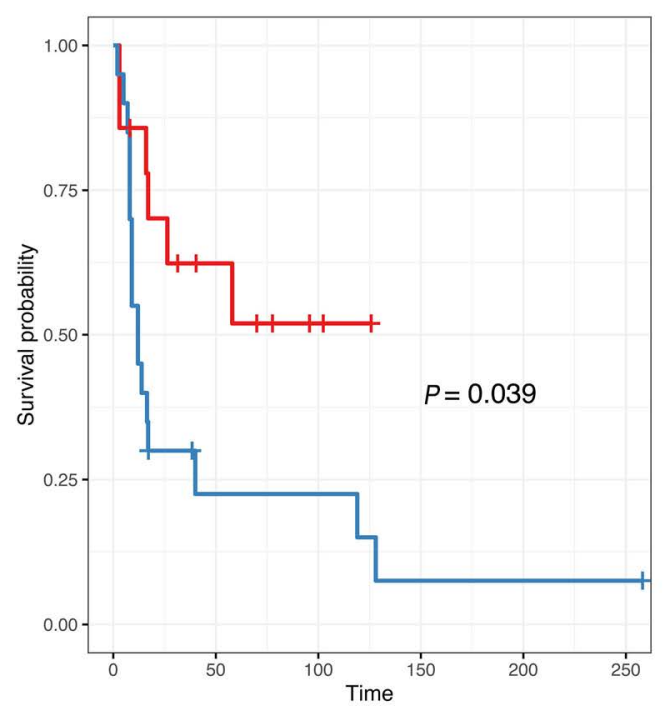

B

Progression-free survival hazard ratio

+ CDKN2A wt

+ CDKN2A loss

+ CDKN2A wt

+ CDKN2A loss

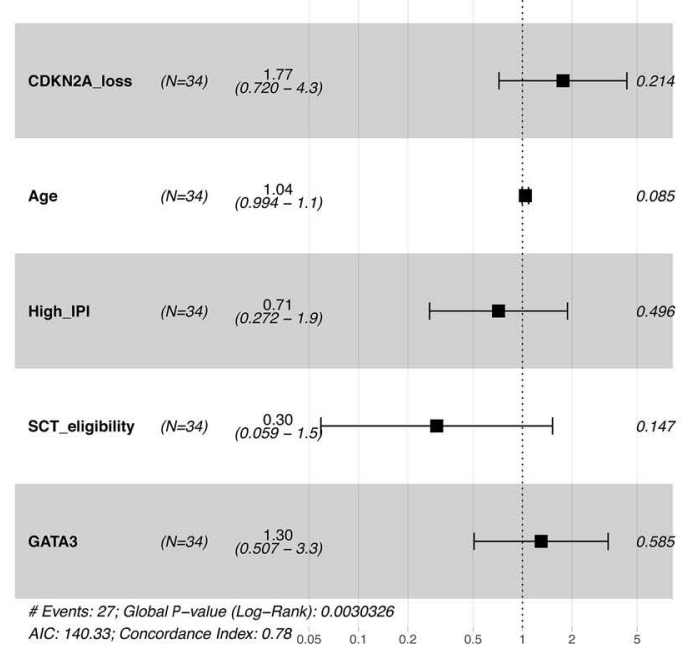

D

\section{Overall survival hazard ratio}
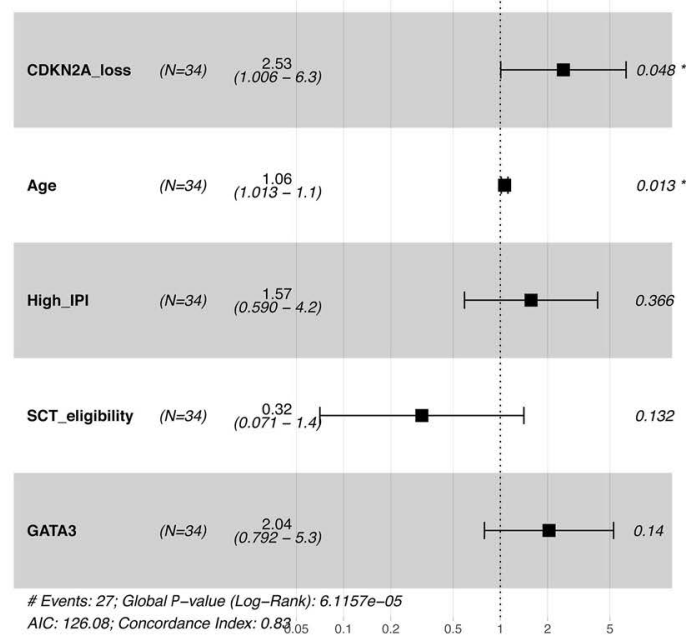

Figure 4. CDKN2A deletion clinical impact. (A and B) Progression-free survival (PFS) and (C and D) overall survival (OS) according to CDKN2A status. (A and C) Kaplan-Meier plots of survival. P-values were estimated using a log-rank test. (B and D) multivariate analysis of survival, performed using Cox regression.

observation in a large validation cohort, showing how other PTCL subtypes did not share these abnormalities, pointing at a specific driver role of CDKN2A in PTCL-NOS pathogenesis. What was most striking though, was that PTEN and CDKN2A co-deletion had a $100 \%$ positive predictive value for the diagnosis of PTCL-NOS, providing the first genetic abnormality that provides absolute specificity for this PTCL subgroup. The integration of these genomic aberrations with point mutations recently reported in distinct subtypes might allow the generation of an accurate diagnostic flow based on NGS as described for other malignancies, ${ }^{42-44}$ potentially able to overcome historical classification difficulties in PTCL.

Importantly, deletions of PTEN, a negative regulator of the PI3K pathway were also enriched in PTCL-NOS. From a mechanistic point of view, this suggests a possible epistatic relationship between the two pathways, and in fact the role of PTEN in T lymphomagenesis has been postulated in vivo. ${ }^{45}$

A distinction between PTCL-NOS cases has recently been proposed, as some cases would show a more complex genomic architecture with frequent CNA involving tumor suppressor genes and worse prognosis, while others would show fewer abnormalities and carry a better prognosis. ${ }^{19,20}$ A correlation between the former group and higher GATA3 expression has also been postulated by some groups, ${ }^{19}$ but not confirmed by others. ${ }^{20}$ High GATA3 expression is itself an adverse prognostic marker in PTCL-NOS. ${ }^{17}$ However, in our series, allelic status of CDKN2A did not correlate with GATA3 expression by IHC. Furthermore, we found that $\mathrm{p} 16$ expression by IHC was a poor predictor of CDKN2A genetic status, likely suggesting that, similarly to other cancers, ${ }^{37}$ non-genetic mechanisms converge towards p16 down-regulation as a 
universal driver of PTCL-NOS. Given the high heterogeneity of the PTCL-NOS subgroup, and the variability of IHC staining of different proteins, further studies will be needed to validate these findings. However, our multivariate analysis of survival confirmed that CDKN2A allelic status was a significant predictor of inferior PFS and OS in PTCL-NOS. Importantly, this was independent of age, IPI, transplant eligibility and GATA3 expression by multivariate analysis. Future studies on larger number of patients will be required to confirm our findings.

While the molecular categorization of PTCL-NOS still remains controversial, our study confirms the utility of WGS in the study of malignancies where recurrent gene mutations are lacking and the classification itself is often ambiguous. Moving from the complexities of WGS, we also show that an old-fashioned FISH test can be of relevance in PTCL-NOS. In the future, the diagnostic value of CDKN2A and PTEN co-deletion, and the prognostic value of CDKN2A deletions will need to be assessed in prospective studies.

\section{Disclosures}

No conflicts of interest to disclose.

\section{Contributions}

PC, FM designed the study, collected and analyzed the data and wrote the paper; $D L, A D, C C, N B$ analyzed the data and wrote the paper; $B R M, J T, J Z, F A, A B, P J C, B D$, and IM analyzed the data; AT, AC, MF and GP performed the IHC and FISH validations; GB, MM and $A C$ collected the data.

\section{Funding}

This work is supported by AIRC (Associazione Italiana Ricerca sul Cancro) and AIL (Associazione Italiana Contro le LeucemieLinfomi e Mieloma ONLUS). This work is supported by the Memorial Sloan Kettering Cancer Center NCI core grant (P30 CA 008748). FM is supported by the American Society of Hematology, the International Myeloma Foundation and The Society of Memorial Sloan Kettering Cancer Center.

\section{References}

1. de Leval L, Gaulard P. Cellular origin of T-cell lymphomas. Blood. 2014;123(19):29092910.

2. Inghirami G, Chan WC, Pileri S, the AIRC 5xMille consortium 'Genetics-driven targeted management of lymphoid malignancies.' Peripheral T-cell and NK cell lymphoproliferative disorders: cell of origin, clinical and pathological implications. Immunol Rev. 2015;263(1):124-159.

3. Maura F, Dodero A, Carniti C, Bolli N. Biology of peripheral $\mathrm{T}$ cell lymphomas not otherwise specified: is something finally happening? Pathogenesis. 2016;3(1):9-18.

4. Swerdlow SH, Campo E, Harris NL, et al., editors. WHO classification of tumours of haematopoietic and lymphoid tissues. Revised 4th edition. Lyon: International Agency for Research on Cancer; 2017. 585 p.

5. Crescenzo R, Abate F, Lasorsa E, et al. Convergent mutations and kinase fusions lead to oncogenic STAT3 activation in anaplastic large cell lymphoma. Cancer Cell. 2015;27(4):516-532.

6. Palomero T, Couronné L, Khiabanian $\mathrm{H}$, et al. Recurrent mutations in epigenetic regulators, RHOA and FYN kinase in peripheral $\mathrm{T}$ cell lymphomas. Nat Genet. 2014;46(2):166170.

7. Sakata-Yanagimoto M, Enami T, Yoshida K, et al. Somatic RHOA mutation in angioimmunoblastic T cell lymphoma. Nat Genet. 2014;46(2):171-175.

8. Yoo HY, Sung MK, Lee SH, et al. A recurrent inactivating mutation in RHOA GTPase in angioimmunoblastic T cell lymphoma. Nat Genet. 2014;46(4):371-375

9. Allen PB, Pro B. Therapy of peripheral T cell lymphoma: focus on nodal subtypes. Curr Oncol Rep. 2020;22(5):44.

10. Lemonnier F, Dupuis J, Sujobert P, et al. Treatment with 5-azacytidine induces a sustained response in patients with angioimmunoblastic T-cell lymphoma. Blood. 2018;132(21):2305-2309.

11. Schatz JH, Horwitz SM, Teruya-Feldstein J, et al. Targeted mutational profiling of peripheral T-cell lymphoma not otherwise specified highlights new mechanisms in a heterogeneous pathogenesis. Leukemia. 2015;29(1):237-241.

12. Abate F, da Silva-Almeida AC, Zairis S, et al.
Activating mutations and translocations in the guanine exchange factor VAV1 in peripheral T-cell lymphomas. Proc Natl Acad Sci U S A. 2017;114(4):764-769.

13. Laginestra MA, Cascione L, Motta G, et al. Whole exome sequencing reveals mutations in FAT1 tumor suppressor gene clinically impacting on peripheral T-cell lymphoma not otherwise specified. Mod Pathol. 2020;33(2):179-187.

14. Corradini P, Vitolo U, Rambaldi A, et al. Intensified chemo-immunotherapy with or without stem cell transplantation in newly diagnosed patients with peripheral T-cell lymphoma. Leukemia. 2014;28(9):18851891.

15. d'Amore F, Leppä S, Silva MG da, et al. Final analysis of the front-line phase III randomized ACT-1 trial in younger patients with systemic peripheral T-cell lymphoma treated with CHOP chemotherapy with or without alemtuzumab and consolidated by autologous hematopoietic stem cell transplant. Blood. 2018;132(Suppl 1):S998-998.

16. Iqbal J, Wright G, Wang C, et al. Gene expression signatures delineate biological and prognostic subgroups in peripheral Tcell lymphoma. Blood. 2014;123(19):29152923.

17. Wang $\mathrm{T}$, Feldman AL, Wada DA, et al. GATA-3 expression identifies a high-risk subset of PTCL, NOS with distinct molecular and clinical features. Blood. 2014;123 (19):3007-3015.

18. Amador C, Greiner TC, Heavican TB, et al Reproducing the molecular subclassification of peripheral T-cell lymphoma-NOS by Immunohistochemistry. 2019;134(24):2159-2170.

19. Heavican TB, Bouska A, Yu J, et al. Genetic drivers of oncogenic pathways in molecular subgroups of peripheral T-cell lymphoma. Blood. 2019;133(15):1664-1676.

20. Watatani Y, Sato Y, Miyoshi $\mathrm{H}$, et al. Molecular heterogeneity in peripheral T-cell lymphoma, not otherwise specified revealed by comprehensive genetic profiling. Leukemia. 2019;33(12):2867-2883.

21. The ICGC/TCGA Pan-Cancer Analysis of Whole Genomes Consortium. Pan-cancer analysis of whole genomes. Nature. 2020;578(7793):82-93

22. Munchel S, Hoang Y, Zhao Y, et al. Targeted or whole genome sequencing of formalin fixed tissue samples: potential applications in cancer genomics. Oncotarget. 2015;6(28): 25943-25961.

23. Maura F, Bolli N, Angelopoulos N, et al. Genomic landscape and chronological reconstruction of driver events in multiple myeloma. Nat Commun. 2019;10(1):3835.

24. Jones D, Raine KM, Davies $\mathrm{H}$, et al. cgpCaVEManWrapper: simple execution of CaVEMan in order to detect somatic single nucleotide variants in NGS data. Curr Protoc Bioinformatics 2016;56:15.10.1-15.10.18.

25. Raine KM, Hinton J, Butler AP, et al cgpPindel: identifying somatically acquired insertion and deletion events from paired end sequencing. Curr Protoc Bioinformatics. 2015:52:15.7.1-15.7.12

26. PCAWG Structural Variation Working Group, PCAWG Consortium, Li Y, et al Patterns of somatic structural variation in human cancer genomes. Nature. 2020;578 (7793):112-121.

27. Alvarez EG, Baez-Ortega A, Zamora J, et al. Pan-cancer analysis of whole genomes identifies driver rearrangements promoted by LINE-1 retrotransposition. Nat Genet. 2020;409(3):860-814.

28. Maura F, Degasperi A, Nadeu F, et al. A practical guide for mutational signature analysis in hematological malignancies. Nat Commun. 2019;10(1):2969.

29. PCAWG Mutational Signatures Working Group, PCAWG Consortium, Alexandrov LB, et al. The repertoire of mutational signatures in human cancer. Nature. 2020;578(7793):94-101.

30. Roberti A, Dobay MP, Bisig B, et al. Type II enteropathy-associated T-cell lymphoma features a unique genomic profile with highly recurrent SETD2 alterations. Nat Commun. 2016;7(1):12602.

31. Wang M, Zhang S, Chuang S-S, et al Angioimmunoblastic $\mathrm{T}$ cell lymphoma: novel molecular insights by mutation profiling. Oncotarget. 2017;8(11):17763-17770.

32. Boi M, Rinaldi A, Kwee I, et al PRDM1/BLIMP1 is commonly inactivated in anaplastic large T-cell lymphoma. Blood. 2013;122(15):2683-2693.

33. Hartmann S, Gesk S, Scholtysik R, et al. High resolution SNP array genomic profiling of peripheral T cell lymphomas, not otherwise specified, identifies a subgroup with chromosomal aberrations affecting the REL 
locus. Br J Haematol. 2010;148(3):402-412.

34. Wang K, Li M, Hadley D, et al. PennCNV: an integrated hidden Markov model designed for high-resolution copy number variation detection in whole-genome SNP genotyping data. Genome Res. 2007;17(11): 1665-1674.

35. Korbel JO, Campbell PJ. Criteria for inference of chromothripsis in cancer genomes. Cell. 2013;152(6):1226-1236.

36. Alhejaily A, Day AG, Feilotter HE, Baetz T, LeBrun DP. Inactivation of the CDKN2A tumor-suppressor gene by deletion or methylation is common at diagnosis in follicular lymphoma and associated with poor clinical outcome. Clin Cancer Res. 2014;20(6):1676-1686.

37. Pasqualucci L, Khiabanian H, Fangazio M, et al. Genetics of follicular lymphoma transformation. Cell Rep. 2014;6(1):130-140.
38. Dodero A, Spina F, Narni F, et al. Allogeneic transplantation following a reduced-intensity conditioning regimen in relapsed/refractory peripheral T-cell lymphomas: long-term remissions and response to donor lymphocyte infusions support the role of a graft-versus-lymphoma effect. Leukemia. 2012;26(3): 520-526.

39. Kataoka K, Nagata Y, Kitanaka A, et al Integrated molecular analysis of adult $\mathrm{T}$ cell leukemia/lymphoma. Nat Genet. 2015;47 (11):1304-1315

40. Reddy A, Zhang J, Davis NS, et al. Genetic and functional drivers of diffuse large $B$ cell lymphoma. Cell. 2017;171(2):481-494.

41. Karube K, Enjuanes A, Dlouhy I, et al Integrating genomic alterations in diffuse large B-cell lymphoma identifies new relevant pathways and potential therapeutic tar- gets. Leukemia. 2018;32(3):675-684

42. Bolli N, Li Y, Sathiaseelan V, et al. A DNA target-enrichment approach to detect mutations, copy number changes and immunoglobulin translocations in multiple myeloma. Blood Cancer J. 2016;6(9):e467.

43. Bolli N, Manes N, McKerrell T, et al. Characterization of gene mutations and copy number changes in acute myeloid leukemia using a rapid target enrichment protocol. Haematologica. 2015;100(2):214-222

44. McKerrell T, Moreno T, Ponstingl H, et al Development and validation of a comprehensive genomic diagnostic tool for myeloid malignancies. Blood. 2016;128(1):e1-9.

45. Liu X, Karnell JL, Yin B, et al. Distinct roles for PTEN in prevention of $\mathrm{T}$ cell lymphoma and autoimmunity in mice. J Clin Invest. 2010;120(7):2497-2507. 